\title{
Coronoid Fractures of the Elbow
}

\author{
Jason Wells, BS and Robert H. Ablove, MD
}

\begin{abstract}
Coronoid fractures of the ulna are relatively uncommon, yet critical injuries to recognize. They often occur in association with elbow dislocations and play an important role in elbow instability. Historic recommendations are to fix all large coronoid fracture fragments, as well as small fracture fragments associated with instability. There is little data regarding management of small coronoid fracture fragments. The coronoid process acts as a bony buttress to prevent posterior dislocation and has three soft tissue insertions which lend stability as well: the anterior joint capsule of the elbow, the brachialis muscle and the medial ulnar collateral ligament. Injured patients often present with swelling, tenderness and limited range of motion. After obtaining a detailed history and performing a careful physical examination, plain radiographs should be obtained. If present, dislocations are reduced and post-reduction stability is assessed. If the elbow is unstable, management usually consists of a combination of bony and soft-tissue repairs often including coronoid process repair. Loss of motion is the most common complication of these injuries. The current recommendation is to repair virtually all coronoid fractures associated with instability.
\end{abstract}

Keywords: Coronoid process fracture, Elbow instability, Elbow injury, Elbow trauma

raumatic elbow injuries, particularly those associated with a dislocation, can lead to elbow instability by damaging not only the bony structures, but also the soft tissues. The coronoid process of the ulna is one of the bony structures that can be fractured and has an important role in the stability of elbows after dislocation.

Coronoid fractures are relatively uncommon injuries occurring in approximately $2 \%$ to $15 \%$ of patients with dislocation. ${ }^{1,2}$ They often occur in an injury, termed the "terrible triad of the elbow,"3,4,5 which involves a posterior or posterolateral elbow dislocation, a radial head fracture, as well as a coronoid process fracture.

Management of coronoid fractures associated with elbow dislocations is complex. Simple dislocations without associated fractures usually respond to closed reduction, brief splinting, and gentle mobilization. Similar treatment can be successful with isolated undisplaced fractures. Associated fractures, such as those involved in the so called "triad injuries" usually require operative management.

\section{Anatomy of the Elbow}

The elbow is a trocho-gynglymoid joint with both rotatory and hinge components. It consists of articulations between the humerus, ulna and radius. The hinge is formed by the trochlea of the humerus and the trochlear notch of the proximal ulna as well as by the radial head and the capitellum. The distal humerus has two fossae that enable maximal flexion and extension. Anteriorly, the coronoid fossa accommodates the coronoid process of the humerus during terminal flexion. Posteriorly, the olecranon fossa accommodates the olecranon process of the ulna during terminal extension. Rotation occurs between the radial head and radial notch of the ulna as well as between the radial head and the capitellum of the distal humerus (figure 1).

The proximal ulna forms a 190 degree arc about the trochlea known as the trochlear notch. The olecranon forms the posterior portion of this notch while the coronoid process forms the anterior portion. The opening of this arc is directed 30 degrees posteriorly and corresponds with the 30 degree anterior rotation of the articular portion of the distal humerus
Received: February 2, 2007
Revised: September 24, 2007
Accepted: December 19, 2007

doi: $10.3121 / \mathrm{cmr} .2008 .753$ 


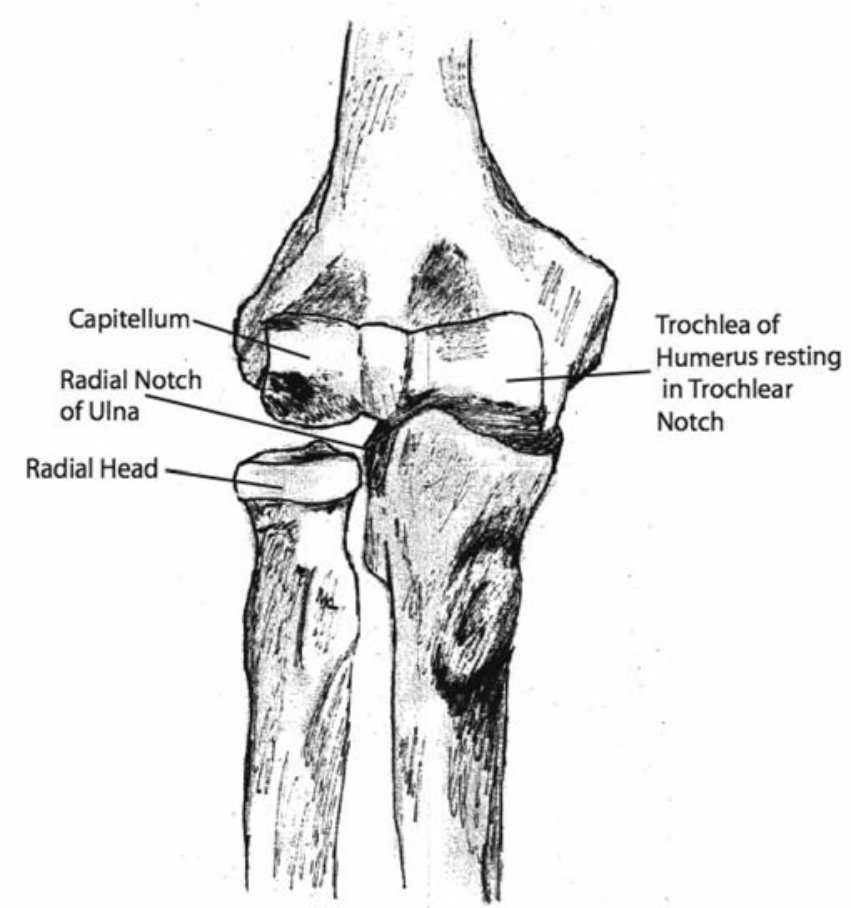

Figure 1. Anterior view of elbow joint articulations and points of rotation.

(relative to the longitudinal axis of the humerus). The coronoid functions as a bony buttress to posterior ulnar displacement. ${ }^{6}$ It receives much of the axial load on the elbow between 60 degrees and 105 degrees of flexion. Cadaveric modeling has demonstrated in extension and axial loading that approximately $40 \%$ of the load is distributed across the ulno-humeral joint.

The coronoid process is also a key stabilizer to varus stress and likely contributes more to stability in extension than in flexion. ${ }^{7}$ The coronoid process has three soft tissue insertions: the anterior joint capsule of the elbow, the brachialis muscle and the medial ulnar collateral ligament (MUCL). 8,9 The anterior capsule inserts very close to the tip, the brachialis inserts anteriorally, distal to the capsule, and the anterior bundle of the MUCL inserts distally and medially on the sublime tubercle of the distal ulna.

The collateral ligaments are important stabilizers of the elbow. The MUCL is the main stabilizer against valgus stress. ${ }^{10,11,12}$ It has also been noted to resist distraction of the elbow joint. ${ }^{12}$ Isolated valgus instability of the elbow can be found in throwing athletes (e.g., baseball, javelin) due to rupture or attenuation of the MUCL. ${ }^{11}$

Morrey and $\mathrm{An}^{13}$ stated the lateral collateral complex varies more than the medial complex, which consists of the radial collateral ligament, the ulnar collateral ligament, the annular ligament, and on occasion, an accessory collateral ligament (figure 2). The lateral ulnar collateral ligament has been shown to be one of the main stabilizers on the lateral side, is taut in both flexion and extension, and has been shown to be deficient in posterolateral rotatory instability of the elbow. ${ }^{2}$ Lateral ulnar collateral ligament deficiency is a major cause of recurrent dislocation and instability.

\section{Mechanism of Injury}

Coronoid fractures usually occur in association with elbow dislocations. Morrey and $\mathrm{An}^{12}$ described elbow dislocation as "usually a high energy episode with severe soft tissue injury," many occurring during sports,, $14-16$ daily activities ${ }^{17}$ or at work. ${ }^{16}$ Major traumas such as motor vehicle accidents can also cause these injuries. ${ }^{15,18}$ The most common cause of an elbow dislocation is a fall on the elbow or outstretched hand from standing height ${ }^{4,14,19}$ or higher. $4,15,18-23$

The specific mechanisms of coronoid fracture (and elbow dislocation) are varied and can involve twisting and flexion, as well as hyperextension. 5,10,22,24,25 When the elbow is axially loaded in extension, force is concentrated on the articular surface of the coronoid process. In large coronoid fractures this anterior buttress is completely disrupted. In twisting flexion injuries, the coronoid process can be sheared off by the trochlea. In hyperextension injuries, the coronoid can fracture via a combination of mechanisms. 1,15,18,26,27 During dislocation, the trochlea can axially load the coronoid process leading to a shear fracture during terminal extension. There may also be an avulsive component with the capsule pulling off the tip of the coronoid.

Anteromedial facet fractures of the coronoid process have been recently described as a distinct type of coronoid fracture caused by a varus posteromedial rotational force. As the elbow is forced into varus, the anteromedial facet is sheared off by the trochlea. 28,29

\section{Coronoid Fracture Types and Associated Injuries}

Three types of coronoid fracture were described by Regan and Morrey: ${ }^{26}$ type I fractures involve the tip of the coronoid, type II fractures involve more than the tip and less than $50 \%$ of the coronoid, and type III fractures involve greater than $50 \%$. There are A and B designations for each type, in which the B indicates associated dislocation. ${ }^{26}$

O'Driscoll et $\mathrm{al}^{28}$ described a new classification system involving 3 fracture types. Type 1 is a tip fracture, type 2 is an anteromedial facet fracture, and type 3 is a fracture through the base of the process.

Historical recommendations have been to fix all Morrey type II and III coronoid fractures as well as any type I fractures associated with instability.1,21,26 However, until recently there has not been a great deal of evidence either describing instability associated with Morrey type I coronoid fractures or supporting the efficacy of their repair. ${ }^{20}$

Josefsson ${ }^{19}$ reported on 4 patients who experienced recurrent instability following an initial dislocation. All had an associated small fragment fracture of coronoid that was not 


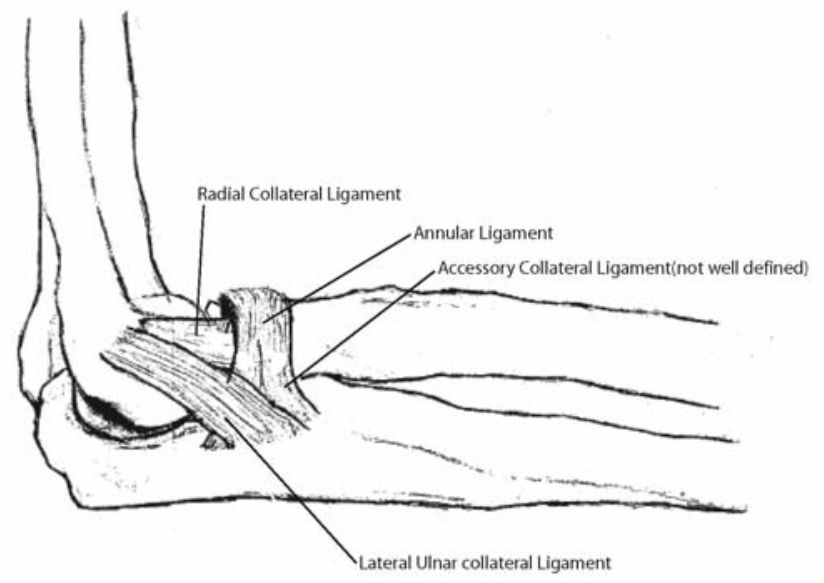

Figure 2. Lateral view of elbow demonstrating ligament complex.

repaired at the time of initial treatment. Terada 20 demonstrated repair of type I fractures can improve stability.

Possible explanations include recent anatomic evidence that noted the capsule usually attaches very close to the tip of the coronoid process. ${ }^{9}$ Clinical evidence reported by Pugh $^{30}$ corroborates that finding, stating that type I injuries usually involve the capsular insertion.

\section{Presentation and Evaluation}

Most patients with coronoid fractures present following elbow dislocations. Elbow dislocation patients often present with a history of a hyperextension or twisting and hyperflexion with a feeling of dislocation followed by a spontaneous reduction.1,30 In unreduced dislocations an obvious deformity is usually present. Patients present in pain with diffuse swelling and tenderness. ${ }^{1,27}$ Tenderness can be multifocal and corresponds with the areas of maximal injury. Range of motion, including flexion, extension and rotation, is limited. Crepitation and anterior-posterior instability is present. Plain radiographs, including at least a posteroanterior, lateral view and radial head view, are necessary to check for any fractures.

After obtaining a detailed history, the next step is performing a physical exam. If upon inspection, the elbow is obviously deformed, defer range of motion testing. Neurovascular testing is paramount. Light touch and 2-point discrimination need to be carefully assessed. Examine for any abnormal or diminished pulses and reassess after any deformity is reduced.

Obtain plain radiographs to check for any fractures or dislocation prior to any manipulation. Manipulative reduction may not be advisable for severely comminuted fractures and/or dislocations. The majority of elbow dislocations can be reduced under adequate analgesia. Radiographs need to be obtained after reduction without any splint in place to confirm both concentric reduction and that there are no intra- articular loose bodies from any associated fractures. Small coronoid fractures are easy to miss and can be hard to distinguish from radial head fractures. ${ }^{31,32}$ In cases where plain radiographs are not definitive, computed tomography (CT) or magnetic resonance imaging (MRI) can be performed. CT offers better bony definition while MRI yields better soft tissue identification.

After reduction, stability needs to be carefully evaluated. This often involves assessment with fluoroscopic assistance under a general anesthetic. Virtually every elbow dislocation should be referred for orthopedic evaluation.

\section{Management}

Most elbows that remain unstable following closed reduction either have significant fractures or severe soft tissue disruption or both. The most commonly associated fractures are coronoid fractures and radial head fractures.

Large coronoid fractures can be rigidly fixed and small or comminuted fractures can be fixed with suturing the bone and anterior capsule to the anterior ulna using pull out sutures or suture anchors. 4,26 The rare small fragments with no soft tissue attachments can be excised.

Operative management of radial head fractures can consist of internal fixation or replacement of fractured radial heads. The degree of comminution determines whether the fracture can be fixed.

Disrupted ligaments should be fixed or reconstructed as well. The lateral ulnar collateral ligament is probably more commonly disrupted than the medial ulnar collateral ligament; both usually avulse from the humerus. If the elbow remains unstable despite all of the above measures, hinged external fixation is necessary.

Early mobilization is desirable since prolonged immobilization (beyond 3-4 weeks) leads to almost universally poor results including persistent stiffness, pain and loss of function. $4,15,18,26,30,32$ The elbow is protected from varus and valgus stress with hinged bracing. The terminal 30 degrees of extension is usually blocked during early rehabilitation for both open and closed treatment. ${ }^{26,30,32} \mathrm{We}$ determine the degree of extension allowed by checking stability under fluoroscopic visualization.

\section{Patient Follow-up and Recommendations}

Follow-up care is a balance between maintaining stability and regaining motion. As splinting is continued and the elbow is gradually mobilized, serial radiographs are necessary to confirm the maintenance of a concentric reduction as well as the possible presence of heterotopic ossification. A true lateral radiograph is the most important confirmatory view.

Therapy plays a central role in mobility recovery. Patients who are slow to recover motion may require either serial 
progressive splints or dynamic splints. Most functional activities can be performed between 30 degrees and 130 degrees of elbow flexion. If mobility recovery is unsatisfactory, patients may require surgical contracture releases. Patients who develop heterotopic ossification may require additional surgery as well.

\section{Outcome and Complications}

There are many long-term complications from both operative and nonoperative treatment of elbow dislocations with and without coronoid fractures. There is not much available data regarding coronoid fractures as an isolated entity. The most common complication of elbow dislocations is loss of range of motion, which has a high correlation with prolonged immobilization. 4,15,18,26,30,32 Loss of terminal extension alone is common and not usually symptomatic. Other complications include osteoarthritis, heterotopic ossification, calcification, instability, pain and parasthesias. ${ }^{1,22,26}$ It is not unusual to see degenerative changes on plain radiographs, but they are not necessarily symptomatic. ${ }^{16}$ Younger patients tend to experience fewer post-dislocation symptoms. ${ }^{16}$

\section{Follow-up}

Close follow-up is necessary to monitor for mobility recovery, maintenance of a concentric reduction and healing of any associated fractures. Patients often require therapy to assist with mobility recovery and patients who do not recover adequate motion may require surgery.

\section{Discussion}

Coronoid fractures rarely occur in isolation and are usually associated with elbow dislocations. The initial evaluation should consist of a careful history, plain radiographs and a thorough physical examination. The presence of a coronoid fracture on plain radiographs, even in the presence of a located joint, may be a harbinger of a serious injury.

Most dislocations should be initially treated with a closed reduction under adequate analgesia. Post-reduction radiographs should be obtained prior to splinting to identify fractures which may not have been evident on pre-reduction films.

The current consensus is to fix virtually all coronoid fractures associated with elbow instability. Large coronoid fractures have a high incidence of elbow instability. Recently, there has been some evidence of instability associated with small coronoid fractures. Small coronoid fractures not associated with elbow instability do not usually require surgical treatment unless they become symptomatic loose bodies. Careful long-term follow-up is necessary to monitor recovery and intervene where necessary.

\section{References}

1. Selesnick FH, Dolitsky B, Haskell SS. Fracture of the coronoid process requiring open reduction with internal fixation. A case report. J Bone Joint Surg Am 1984;66:1304-1306.
2. Morrey BF. The elbow and its disorders. 3rd ed. Philadelphia, PA: W.B. Saunders Company; 2000.

3. Hotchkiss RN. Fractures and dislocations of the elbow. In: Rockwood CA, Green DP, eds. Rockwood and Green'sfractures in adults. Vol 1. 4th ed. Philadelphia, PA: Lippincott Williams \& Wilkins; 1996:929-1024.

4. Ring D, Jupiter JB, Zilberfarb J. Posterior dislocation of the elbow with fractures of the radial head and coronoid. J Bone Joint Surg Am 2002;84-A:547-551.

5. Doornberg JN, van Duijn J, Ring D. Coronoid fracture height in terrible-triad injuries. J Hand Surg Am 2006;31:794-797.

6. Closkey RF, Goode JR, Kirschenbaum D, Cody RP. The role of the coronoid process in elbow stability. A biomechanical analysis of axial loading. J Bone Joint Surg Am 2000; 82-A:1749-1753.

7. Hull JR, Owen JR, Fern SE, Wayne JS, Boardman ND 3rd. Role of the coronoid process in varus osteoarticular stability of the elbow. J Shoulder Elbow Surg 2005;14:441-446.

8. Cage DJ, Abrams RA, Callahan JJ, Botte MJ. Soft tissue attachments of the ulnar coronoid process. An anatomic study with radiographic correlation. Clin Orthop Relat Res 1995 Nov;(320):154-158.

9. Ablove RH, Moy OJ, Howard C, Peimer CA, S'Doia S. Ulnar coronoid process anatomy: possible implications for elbow instability. Clin Orthop Relat Res 2006 Aug;(449):259-261.

10. O’ Driscoll SW, Morrey BF, Korinek S, An KN. Elbow subluxation and dislocation. A spectrum of instability. Clin Orthop Relat Res 1992 Jul;(280):186-197.

11. Conway JE, Jobe FW, Glousman RE, Pink M. Medial instability of the elbow in throwing athletes. Treatment by repair or reconstruction of the ulnar collateral ligament. J Bone Joint Surg Am 1992;74:67-83.

12. Morrey BF, An KN. Articular and ligamentous contributions to the stability of the elbow joint. Am J Sports Med 1983;11:315-319.

13. Morrey BF, An KN. Functional anatomy of the ligaments of the elbow. Clin Orthop Relat Res 1985 Dec;(201):84-90.

14. Josefsson PO, Johnell O, Wendeberg B. Ligamentous injuries in dislocations of the elbow joint. Clin Orthop Relat Res 1987 Aug;(221):221-225.

15. Mehlhoff TL, Noble PC, Bennett JB, Tullos HS. Simple dislocation of the elbow in the adult. Results after closed treatment. J Bone Joint Surg Am 1988;70:244-249.

16. Josefsson PO, Johnell O, Gentz CF. Long-term sequelae of simple dislocation of the elbow. J Bone Joint Surg Am 1984;66:927-930.

17. Habernek H, Ortner F. The influence of anatomic factors in elbow joint dislocation. Clin Orthop Relat Res 1992 Jan;(274):226-230.

18. Cobb TK, Morrey BF. Use of distraction arthroplasty in unstable fracture dislocations of the elbow. Clin Orthop Relat Res 1995 Mar;(312):201-210.

19. Josefsson PO, Gentz CF, Johnell O, Wendeberg B. Dislocations of the elbow and intraarticular fractures. Clin Orthop Relat Res 1989 Sep;(246):126-130.

20. Terada N, Yamada H, Seki T, Urabe T, Takayama S. The importance of reducing small fractures of the coronoid process in the treatment of unstable elbow dislocation. J Shoulder Elbow Surg 2000;9:344-346.

21. Hanks GA, Kottmeier SA. Isolated fracture of the coronoid process of the ulna: a case report and review of the literature. J Orthop Trauma 1990;4:193-196.

22. Doria A, Gil E, Delgado E, Alonso-Llames M. Recurrent dislocation of the elbow. Int Orthop 1990;14:41-45.

23. Seijas R, Joshi N, Hern·ndez A, Catal·n JM, Flores X, Nieto JM. Terrible triad of the elbow-role of the coronoid process: a case report. J Orthop Surg (Hong Kong) 2005;13:296-299. 
24. Doornberg JN, Ring D. Coronoid fracture patterns. J Hand Surgery [Am] 2006;31:45-52.

25. Sanchez-Sotelo J, O'Driscoll SW, Morrey BF. Anteromedial fracture of the coronoid process of the ulna. J Shoulder Elbow Surg 2006;15:e5-8. Erratum in: J Shoulder Elbow Surg 2007;16:127.

26. Regan W, Morrey B. Fractures of the coronoid process of the ulna. J Bone Joint Surg Am 1989;71:1348-1354.

27. Gadgil A, Roach R, Neal N, Maffulli N. Isolated avulsion fracture of the coronoid process requiring open reduction in a paediatric patient: a case report. Acta Orthop Belg 2002;68:396-398.

28. O'Driscoll SW, Jupiter JB, Cohen MS, Ring D, McKee MD. Difficult elbow fractures: pearls and pitfalls. Instr Course Lect 2003;52:113-134.

29. Doornberg JN, Ring DC. Fracture of the anteromedial facet of the coronoid process. J Bone Joint Surg Am 2006;88:2216-2224.

30. Pugh DM, Wild LM, Schemitsch EH, King GJ, McKee MD. Standard surgical protocol to treat elbow dislocations with radial head and coronoid fractures. J Bone Joint Surg Am 2004;86-A:1122-1130.

31. McGinley JC, Roach N, Hopgood BC, Kozin SH. Nondisplaced elbow fractures: A commonly occurring and difficult diagnosis. Am J Emerg Med 2006;24:560-566.

32. McKee MD, Pugh DM, Wild LM, Schemitsch EH, King GJ. Standard surgical protocol to treat elbow dislocations with radial head and coronoid fractures. Surgical technique. J Bone Joint Surg Am 2005;87 Suppl 1:22-32.

\section{Author Affiliations}

Jason Wells, BS

University of Wisconsin School of Medicine

and Public Health

Department of Orthopedics and Rehabilitation

K4/7 Clinical Science Center

600 Highland Avenue

Madison, WI 53792-7375

Robert H. Ablove, $M D$

Assistant Professor

Chief Hand Surgery Service

University of Wisconsin School of Medicine

and Public Health

Department of Orthopedics and Rehabilitation

K4/7 Clinical Science Center

600 Highland Avenue

Madison, WI 53792-7375 